\title{
Technologies éducatives et apprentissages : l'histoire de TECFA, une unité de recherche et d'enseignement de l'Université de Genève Entretien avec Daniel Peraya
}

\section{Educational technologies and learning: the history of TECFA, a research and teaching unit in the University of Geneva - Interview with Daniel Peraya}

\section{Tecnologías educativas y aprendizaje: la historia de TECFA, una unidad de investigación y enseñanza de la Universidad de Ginebra - Entrevista con Daniel Peraya}

Viviane Glikman, enseignante-chercheuse

Membre du GEHFA (Groupe d'études - histoire de la formation des adultes), France viviane.glikman@free.fr

Cathia Papi, professeure

Université TÉLUQ, Canada

cathia.papi@teluq.ca

RÉSUMÉ

Chercheur renommé dans le domaine des technologies éducatives, Daniel Peraya parle dans cet entretien de l'histoire de TECFA (Technologies de formation et apprentissage), une unité de recherche et d'enseignement de l'Université de Genève qui a joué un rôle important dans de nombreux projets de formation hybride et à distance en Suisse, en Europe et dans les pays du Sud. II explique comment cette unité a été créée et a évolué en termes de recherche et d'enseignement et offre ainsi un éclairage sur la manière dont les nombreuses collaborations 
avec des partenaires ou financeurs suisses, européens et internationaux ont permis à TECFA d'impulser de nouveaux programmes et de favoriser le développement de la formation à distance, devenant ainsi un centre de référence dans le domaine.

Mots-clés : dispositif hybride, environnement d'apprentissage, technologies de l'information et de la communication, usages éducatifs des médias, recherche internationale

\section{ABSTRACT}

In this interview, Daniel Peraya, a renowned researcher in the field of educational technologies, talks about the history of TECFA (Technologies de formation et apprentissage), a research and teaching unit at the University of Geneva that has played an essential role in many hybrid and distance learning projects in Switzerland, Europe and the South. He explains how this unit was created and has evolved in terms of research and teaching, and thus sheds light on how the numerous collaborations with Swiss, European and international partners and funders have enabled TECFA to initiate new programs and promote the development of distance learning, becoming a reference center in the field.

Keywords: hybrid system, learning environment, information and communication technologies, educational uses of media, international research

RESUMEN

En esta entrevista, Daniel Peraya, reconocido investigador en el campo de las tecnologías educativas, habla de la historia de TECFA (Technologies de formation et apprentissage), una unidad de investigación y enseñanza de la Universidad de Ginebra que ha desempeñado un importante papel en numerosos proyectos de aprendizaje híbrido y a distancia en Suiza, Europa y en los países del Sur. Explica cómo se creó y ha evolucionado esta unidad, en términos de investigación y de enseñanza. Además, arroja luz sobre cómo las numerosas colaboraciones con socios y financiadores suizos, europeos e internacionales han permitido a TECFA iniciar nuevos programas y promover el desarrollo de la enseñanza a distancia, convirtiéndose así en un centro de referencia en este campo.

Palabras clave: dispositivo híbrido, entorno de aprendizaje, tecnologías de la comunicación y de la educación, usos educativos de los medios de comunicación, investigación internacional

Daniel Peraya est professeur honoraire à l'unité de recherche et d'enseignement Technologies de formation et apprentissage (TECFA) de la Faculté de psychologie et des sciences de l'éducation (FPSE) à l'Université de Genève. Ses recherches et ses enseignements portent sur la communication éducative médiatisée, plus particulièrement dans le cadre des systèmes de formation entièrement ou partiellement à distance aux niveaux supérieur et universitaire. II a participé à de nombreux projets de recherche dans ce domaine, notamment au niveau européen, a publié un grand nombre d'articles et plusieurs ouvrages sur ce thème et a contribué à faire de TECFA un acteur important dans l'histoire des formations hybrides et à distance. 
VIVIANE GLIKMAN et CATHIA PAPI : L'unité Technologies de formation et apprentissage de l'Université de Genève a joué un rôle important dans la recherche et le développement des usages du numérique dans la formation à distance notamment, mais pas uniquement au niveau européen. Daniel, pourrais-tu nous parler de la création de cette unité et de ses objectifs?

DANIEL PERAYA: TECFA' est née en 1989 d'une initiative d'Edouard Bayer, le doyen de la Faculté de psychologie et des sciences de l'éducation (FPSE), en réponse à un appel à projets innovants dans le cadre du plan de développement quadriennal (1989-1991). Le projet institutionnel était de regrouper et de fédérer, autour d'un projet scientifique et dans une entité propre, des acteurs, leurs initiatives et leurs actions dispersés à travers diverses instances universitaires. Le projet scientifique, quant à lui, consistait à concevoir, à analyser et à évaluer des environnements d'apprentissage instrumentés par l'informatique et les technologies de l'information et de la communication. II s'agissait plus particulièrement d'analyser les effets de ces environnements et de ces dispositifs sur le comportement et l'apprentissage humains des apprenants ainsi que des autres utilisateurs. Les profils des membres de l'unité illustrent bien la volonté de réunir ces deux courants et leurs objets particuliers dans une approche interdisciplinaire, mais aussi dans une structure d'enseignement et de recherche propre. Celle-ci se trouvait renforcée par la structure facultaire, TECFA se trouvant à la croisée des sections de psychologie et des sciences de l'éducation, ce qui s'est révélé une richesse.

VIVIANE GLIKMAN et CATHIA PAPI : Quels étaient alors les profils des membres de l'unité?

DANIEL PERAYA: TECFA a été cofondée par deux personnes : Patrick Mendelsohn, un psychologue grenoblois spécialisé en informatique scolaire, chercheur au CNRS et Daniel Schneider, issu des sciences économiques et sociales, mais passionné par l'informatique et l'intelligence artificielle (IA). Daniel a joué un rôle très important dans les choix et le développement technologiques de TECFA : il a toujours eu une vision prospective extrêmement claire qui a permis à l'unité de se trouver à la pointe de l'innovation. II a, par exemple, mis en place pour TECFA l'un des premiers serveurs Web de Suisse, le site Web de TECFA à cette époque. II a aussi développé l'EduTec Wiki² de TECFA.

En 1990, deux autres personnes ont rejoint TECFA : Pierre Dillenbourg, un ancien instituteur licencié en sciences de l'éducation qui avait pour centre d'intérêt les environnements d'apprentissage informatisés exploitant les techniques de l'intelligence artificielle et qui a fait sa thèse en informatique en 1993, et moimême. Docteur en sciences de l'information et de la communication, j'avais travaillé à l'École normale supérieure, à l'Université de Dakar et à la Faculté de psychologie et des sciences de l'éducation où j'avais assuré des cours de "pédagogie audiovisuelle " (c'était le terme encore en usage à l'époque) dont l'orientation s'inscrivait clairement dans l'approche communicationnelle des médias dans leurs usages éducatifs. J'avais aussi mené, pour le Département de l'instruction publique, une étude préliminaire à une proposition de loi visant à créer une maturité ${ }^{3}$ à distance dans le canton de Genève.

\footnotetext{
${ }^{1}$ Merci à Mireille Bétancourt qui m’a rappelé quelques éléments historiques oubliés.

${ }^{2}$ http ://edutechwiki.unige.ch/fr/Accueil

${ }^{3}$ La « maturité » est un certificat de fin d'études secondaires en Suisse, comme d'ailleurs en Belgique.
} 
VIVIANE GLIKMAN et CATHIA PAPI : Comment l'unité a-t-elle évolué par la suite?

DANIEL PERAYA : Avec le temps, bien sûr, TECFA s'est développée. À l'occasion de son dixième anniversaire, elle avait remis à son comité scientifique son Rapport d'activités 1989-1999. Le Web a 10 ans, nous aussi... (TECFA, 1999). Après deux périodes de probation, cette date consacre la reconnaissance définitive de l'existence de TECFA, sous l'égide du doyen de l'université. Ses activités se sont développées avec, par exemple, la création récente du CeLEN, Conseil en eLearning et Éducation Numérique ${ }^{4}$, une entité de service qui accueille des contrats de recherche et de formation qui fournissent des budgets pour les chercheurs sur fonds externes.

Il y a eu des changements et des remaniements internes, des départs volontaires (Patrick Mendelsohn, Pierre Dillenbourg), des départs à la retraite (le mien en 2014 et récemment celui de Daniel Schneider) et bien sûr de nombreux recrutements qui ont eu pour conséquence de renforcer les axes de recherche ou d'en ouvrir d'autres. Par exemple, Mireille Bétrancourt, qui a succédé à Patrick Mendelsohn, a renforcé le développement de la psychologie cognitive et expérimentale au sein de TECFA; François Lombard, enseignant de biologie dans l'enseignement secondaire, a apporté ses compétences et son expérience professionnelle dans l'intégration des technologies à l'enseignement, particulièrement de la biologie.

TECFA est restée une petite équipe, mais comme elle est très active et qu'elle jouissait à l'époque déjà d'une très bonne reconnaissance scientifique, elle a obtenu, et obtient encore, de très nombreux financements de recherche publics suisses ou européens et même, parfois, apportés par des partenariats avec des fondations ou des entreprises privées. Nous étions donc en permanence une vingtaine de collaborateurs et de collaboratrices dont la grande majorité était engagée sur des fonds externes. Et la situation ne doit pas avoir changé depuis lors.

Puisque le périmètre du champ couvert par TECFA, les "technologies éducatives ", est large, les perspectives, les approches et les objets de recherche se sont diversifiés en fonction de l'évolution du domaine, mais aussi des différents financements obtenus et donc des opportunités : apprentissage multimédia interactif, oculométrie (eye tracking), écriture collaborative et argumentative, ergonomie des systèmes d'information complexes, intégration des technologies en situation d'enseignement et d'apprentissage, jeu, récit interactif et apprentissage, design digital, analytique de l'apprentissage, soutien, processus d'autorégulation, dispositifs de formation et de communications médiatisées entièrement ou partiellement à distance, motivation et persistance des étudiants en formation à distance, etc. (voir le site de TECFA ${ }^{5}$ et les pages des collègues).

VIVIANE GLIKMAN et CATHIA PAPI : Peut-on dire que TECFA, depuis sa création, a joué un rôle dans le développement de la formation à distance et de la formation hybride en Suisse, voire en Europe? Et si oui, lequel?

DANIEL PERAYA : Sans hésiter, oui. Ainsi, en plus de l'étude de faisabilité sur le développement d'une maturité à distance dans le canton dont j'ai déjà parlé, j'ai aussi été chargé par l'OFES (Office fédéral de l'éducation et de la science) d'un premier rapport sur l'état des ressources disponibles pour le développement de la formation à distance en Suisse (1990). Ce rapport, mis à jour en 1996, a servi de base au projet de loi qui a permis la création du programme Campus virtuel suisse (CVS, 2000-2008)6.

\footnotetext{
${ }^{4}$ https ://tecfa.unige.ch/celen/

${ }^{5}$ http ://tecfa.unige.ch/fr/

${ }^{6}$ http ://www.virtualcampus.ch/displaya990.html
} 
Entre-temps, j'ai participé à la création et au comité de pilotage du Centre romand d'enseignement à distance (CRED) entre 1995 et 2001. De cette initiative, après de nombreuses péripéties, est née I'UniDistance ${ }^{7}$ avec laquelle TECFA entretient des liens. Gaëlle Molinari, par exemple, se partage entre TECFA et I'UniDistance.

Le Campus virtuel suisse était un programme fédéral qui visait à développer le potentiel des technologies de l'information et de communication dans leurs usages éducatifs au sein des universités, des hautes écoles et des deux écoles polytechniques fédérales. II faisait partie d'un programme plus vaste destiné à favoriser la société de l'information. Le programme a connu deux phases : la phase initiale (2000-2004) et celle de consolidation (2004-2008). Les projets, pour être financés, devaient réunir un partenariat interuniversitaire et bilingue. Si l'un des objectifs du programme était de positionner la Suisse sur le marché de la formation à distance, l'une de ses plus grandes réussites a été la sensibilisation des enseignants à la pédagogie universitaire et à l'ingénierie pédagogique autant qu'à la diffusion de la culture de la formation à distance, de la formation hybride et de l'intégration des technologies aux activités d'enseignement et d'apprentissage. La direction du CVS a notamment confié à TECFA le mandat d'accompagner les projets coordonnés par une université francophone. Dans cette perspective, Jacques Viens, que j'ai recruté pour accomplir cette mission et avec qui j'ai travaillé durant toute la durée du programme, s'est notamment employé à construire, avec les collègues porteurs de projets, une approche anthropologique plutôt que technocentrée.

Le programme CVS a sans conteste joué un rôle majeur dans le développement de la formation entièrement ou partiellement à distance en Suisse. Mais tous les projets menés sous les auspices de l'OFES, du FNS (Fonds national suisse) ou de l'AUF (Agence universitaire de la Francophonie) ont largement contribué à ce mouvement en Suisse, dans l'Union européenne comme dans les pays francophones du Sud. Ils ont aussi contribué à structurer et à développer un domaine de recherche, des communautés scientifiques ainsi que des communautés d'intérêt et de pratique.

\section{VIVIANE GLIKMAN et CATHIA PAPI : Quelles étaient les activités de formation de TECFA?}

DANIEL PERAYA : Les formations organisées par TECFA ont évolué : intervention dans le cursus de psychologie et des sciences de l'éducation; mise en œuvre du diplôme propre STAF ${ }^{8}$ (1994), premier dispositif hybride de formation en Suisse; participation à la licence « mention enseignant » de la section des sciences de l'éducation, etc. On peut aussi évoquer, entre autres, de nombreuses participations à des actions de formation continue, notamment pour les enseignants genevois. Mais l'essentiel n'est pas, bien sûr, d'aligner les formations assurées par TECFA, mais d'analyser leur originalité et leurs apports dans le champ.

Les premiers enseignements au sein de la FPSE ont été ceux de Mireille Bétrancourt sur les environnements informatiques et le mien, consacré aux dispositifs médiatiques dans leurs usages éducatifs. II s'agit de cours présentiels relativement classiques dans la mesure où s'imposait déjà la nécessité d'une pédagogie par projet et de former aux technologies par les technologies. Ces deux principes ont fondé toute l'approche pédagogique de TECFA. Une autre remarque importante porte sur les deux champs couverts par TECFA à partir d'approches théoriques différentes, bien que complémentaires. Je dirais que les cadres de l'activité instrumentée et celui des dispositifs de formation et de communication médiatisées ont contribué à relier les approches et à mettre en évidence leurs points

\footnotetext{
${ }^{7}$ https://unidistance.ch/

${ }^{8}$ Diplôme « post-grade » (c'est-à-dire de $3^{\mathrm{e}}$ cycle universitaire) en sciences et technologies de l'apprentissage et de la formation.
} 
communs. Cette dualité entre médias (M), images (I) et TIC ainsi que la volonté de les rapprocher étaient d'ailleurs au cœur de la formation « F3MITIC » destinée, de 2001 à 2007, au personnel de l'enseignement obligatoire, formation complémentaire de formateurs de formateurs qui avait notamment pour objectif de créer des ponts entre ces domaines et de promouvoir une littéracie commune, en tout cas complémentaire. Sous l'impulsion de TECFA, membre du Comité de pilotage de la formation, la pédagogie de projet, l'apprentissage des technologies et des médias par leur pratique, la collaboration entre pairs, l'alternance entre travail individuel et par groupe, enfin l'hybridation du dispositif ont inspiré toute l'ingénierie de conception de cette formation.

L'hybridation des dispositifs de formation a été l'une des contributions majeures portées par TECFA. Les premiers cours que nous donnions en première et en deuxième années du programme de la FPSE étaient les deux seuls enseignements relatifs aux technologies éducatives de tout le cursus. Aussi, notre volonté a-t-elle visé à renforcer le domaine par la création d'un diplôme de $3^{\mathrm{e}}$ cycle en technologies éducatives. Le diplôme STAF a donc été créé en 1994 et apportait deux innovations essentielles : le format hybride de la formation et son objet. À l'époque, il n'existait en effet aucune formation hybride, pas plus que de diplôme francophone de $3^{\mathrm{e}}$ cycle dans le domaine. Le mode d'organisation hybride nous est apparu comme une nécessité pour plusieurs raisons. Étant donné la « rareté » de notre formation sur le marché, nous espérions recruter des participants dans une zone géographique bien plus étendue que le bassin genevois. De plus, comme il s'agissait d'une formation de $3^{e}$ cycle, nous visions un public de professionnels (enseignants, responsables de formations, concepteurs et designers pédagogiques, etc.), donc des personnes déjà engagées dans une carrière professionnelle. Pour ces deux raisons, un format présentiel classique était bien évidemment irréalisable.

L'organisation de la formation comportait six périodes de cinq semaines, dont une en présence et quatre encadrées à distance à travers un environnement de travail virtuel, un campus virtuel. Pour chaque cours, chacune des six périodes se terminait par la remise d'un projet individuel ou de groupe. Mais le choix de l'hybridation ne concernait pas seulement le mode d'organisation de la formation. II a touché tous les aspects de celle-ci : une véritable complémentarité entre les activités présentielles et à distance, un environnement numérique de travail - le campus virtuel - constituant un espace commun interreliant les activités présentes et distantes, des ressources médiatisées scénarisées et adaptées aux particularités de la distance, un processus d'encadrement et de soutien des apprenants durant les périodes de travail à distance. De plus, la collaboration, les travaux de groupes en alternance avec les travaux individuels et le développement d'environnements numériques d'apprentissage favorisaient les interactions entre pairs. On le voit, les principes pédagogiques évoqués ci-dessus se sont largement étoffés. Ce sont eux qui ont guidé toutes les formations que nous avons conçues et réalisées : dispositifs hybrides de formation, certificats de formation continue, formations à distance (UTICEF-ACREDITÉ) et son adaptation avec la Confédération et un partenaire valaisan (Projet CoseLearn, 2005-2007). Nous avons aussi testé un dispositif expérimental de « double hybridation », MALTT@DIS (Bernasconi, 2009). Il s'agissait d'intégrer au diplôme MALTT quelques étudiants entièrement à distance qui participaient par visioconférence aux activités des semaines présentielles. Les difficultés de connexion, de communication, mais aussi de scénarisation étaient importantes et nous n'avons pas poursuivi dans cette voie. Cette expérimentation aurait pu mener à de véritables formes de comodalité telles qu'elles se pratiquent aujourd'hui à l'Université Laval.

Je voudrais insister sur une dimension : la scénarisation et l'encadrement proactif des activités réalisées à distance par les apprenants. Dans la recherche européenne Hy-Sup ${ }^{9}$ (2010-2012), nous avons établi une taxonomie des dispositifs hybrides de formation (Peraya et Peltier, 2012; Lebrun et al., 2014). Celle-ci

${ }^{9}$ Voir http ://prac-hysup.univ-lyon1.fr/ 
montrait une distinction nette entre les dispositifs centrés sur l'enseignement et ceux centrés sur l'apprentissage. La principale caractéristique qui permet d'identifier ces deux grandes familles de dispositifs hybrides de formation est justement la scénarisation des activités à distance. Cet aspect, pour moi essentiel, n'est pas sans conséquence dans les mesures d'urgence prises à la suite de la situation que nous connaissons depuis mars 2020 : il ne suffit pas de mettre des ressources en ligne pour mettre en œuvre une formation à distance. Ceux et celles qui pensent que leur rôle s'arrête à la mise en ligne, prétendant que l'appropriation des ressources et finalement l'apprentissage sont de la responsabilité exclusive de l'apprenant font, de mon point de vue, une grave erreur.

\section{VIVIANE GLIKMAN et CATHIA PAPI : Qu'entends-tu par cette notion de campus virtuel? II semble que l'on y recourt plus rarement aujourd'hui.}

DANIEL PERAYA : Du point de vue technologique, au début du diplôme STAF, nous nous sommes appuyés sur le serveur Web et sur le site de TECFA, mais qui n'était encore qu'un serveur de pages, ce qui, avec le recul, paraît aujourd'hui une fonctionnalité assez basique, même si l'on constate que ce que j'appelle la pratique du passe-plat - le dépôt de fichiers par l'enseignant dans l'environnement et leur consultation par les apprenants - comme seul usage de l'environnement est loin d'avoir disparu. Les technologies ont évolué et le Web est devenu interactif; en 1996, nous avons créé notre premier véritable campus virtuel et notre expertise nous a permis par exemple de créer le campus virtuel du projet européen Learn-Nett ${ }^{10}$ (1998-2000) (Joye, Deschryver et Peraya, 2003).

La notion de campus virtuel est intéressante lorsque l'on analyse les formes d'environnements concrets auxquels elle a donné naissance, ainsi que leur évolution. Avec les premières technologies du Web interactif, il est devenu possible d'insérer dans les pages des applications réellement interactives. On avait certes connu auparavant de nombreux dispositifs technologiques basés notamment sur la CMC (Computer Mediated Communication) ou sur le CAL (Computer Aided Learning) (Lifländer, 1993) que j'avais nommés, avec Anne-Sophie Collard, des « protocampus ».

Nous définissions alors un campus virtuel comme « un environnement entièrement dédié à l'enseignement et à l'apprentissage, à la formation supérieure et universitaire. C'est aussi un environnement dynamique basé sur les technologies du Web - Web-based learning environment - qui médiatise l'ensemble des dimensions ou fonctions générales de tout système de formation et met à disposition les outils informatiques - les services - correspondants " (Peraya et Collard, 2008, p. 61). II s'agissait donc d'un espace clos, fermé ${ }^{11}$ (son accès est conditionné par une autorisation institutionnelle, un mot de passe), construit à partir d'une métaphore cognitive, celle de l'espace, qui prend forme dans une représentation linguistique et ensuite graphique. La métaphore spatiale constitue un cadre d'interprétation de cet " espace » et s'exprime à travers tous les termes utilisés: campus, environnement, salle, classe, bibliothèque, etc. qui renvoient au champ sémantique de l'espace et de la spatialité. Ensuite, certains de ces campus virtuels ont fait l'objet de représentations graphiques en deux ou en trois dimensions, comme les premiers campus virtuels de la formation UTICEF. II y a même eu des expérimentations de classes virtuelles dans l'environnement immersif Second life ou dans un environnement immersif et interactif « en images virtuelles » (Lombardi, 2007). Après avoir connu un certain engouement, ces expérimentations ont été progressivement abandonnées pour de multiples raisons (lourdeur de l'ergonomie des environnements, utilité limitée aux novices dans leurs premières prises de contact avec l'environnement,

\footnotetext{
${ }^{10}$ Learn-Nett permet à des étudiants de différentes universités européennes de s'initier aux NTIC à travers un apprentissage par projet. Ce dispositif est basé sur le tutorat et la collaboration à distance. Voir https ://tecfa.unige.ch/proj/learnett/

${ }^{11}$ On y verra sans doute un rapprochement possible avec l'archétype de la forme scolaire telle que l'a définie Vincent $(1980,2008)$.
} 
trop de fonctionnalités sous-utilisées, etc.). Pourtant l'utilisation des environnements immersifs semble avoir suscité un regain d'intérêt durant cette crise sanitaire, notamment pour la possibilité d'y recréer des relations et des liens sociaux « vivants ».

Le second aspect que je voudrais souligner à propos des campus virtuels et des environnements numériques de travail est l'abandon progressif de ces environnements institutionnels par les étudiants à la suite de la multiplication des applications grand public et avec la généralisation de l'usage de l'iPhone au milieu des années 2010. Les applications qu'utilisent nos étudiants dans leur vie quotidienne présentent pour eux de nombreux avantages: elles sont plus légères, paramétrables et personnalisables par l'utilisateur en fonction de ses habitudes, de ses usages et de ses besoins particuliers. Avec Philippe Bonfils nous avons étudié ce phénomène entre 2010 et 2014 auprès des étudiants de l'UFR Ingémédia ${ }^{12}$ à l'Université de Toulon (Bonfils et Peraya, 2011; Peraya et Bonfils, 2012). L'intention initiale sous-jacente à la conception des campus virtuels visait à regrouper des dispositifs éclatés dans un lieu unique. Elle semble avoir cédé le pas à une vision plus ouverte et à nouveau plus disparate des dispositifs technologiques sous la pression des usages privés et domestiques. De ce mouvement de bascule est né le concept d'« environnement personnel d'apprentissage » développé notamment par Attwell (2006).

\section{VIVIANE GLIKMAN et CATHIA PAPI : Tu disais que TECFA avait développé des formations entièrement à distance?}

DANIEL PERAYA : C'est vrai et c'est important. J'ai participé à l'articulation, dès les années 1991 et 1992 , à la conception et à la mise en place d'une formation entièrement à distance par la Faculté de théologie protestante. Ce projet était extrêmement important, car le public de cette formation est peu nombreux et géographiquement très dispersé à travers le monde. La formation à distance était l'unique solution pour assurer à la Faculté un recrutement suffisant. Cela dit, ce premier projet radicalement innovant dans le contexte de l'Université de Genève a permis à TECFA d'asseoir et de faire connaître son expertise au sein de l'Université.

De la formation UTICEF-ACREDITÉ, je ne soulignerai que quelques aspects particuliers. Cette formation entièrement à distance est destinée à des collègues universitaires des pays de la Francophonie, essentiellement dans les pays du Sud désireux d'acquérir de nouvelles compétences dans l'intégration des technologies dans leurs enseignements (Peraya, Depover et Jaillet, 2013). C'est donc une formation professionnalisante basée sur les principes pédagogiques de nos autres formations. Le travail de fin d'études consistait en la conception, le développement, la mise en œuvre expérimentale et l'évaluation d'un projet de formation à taille réelle dans leur contexte institutionnel. Je considère que cette formation a été un succès pour deux raisons au moins. Premièrement, nous avons formé près de cinq cents collègues depuis 2000. Ceux-ci soit ont conservé leurs fonctions d'enseignants-chercheurs, mais en ayant bénéficié d'un véritable développement professionnel, soit sont devenus responsables d'un service de conception de dispositifs hybrides ou à distance ou de soutien technopédagogique dans leur institution. II s'agit bien d'un projet de coopération nord-sud ayant donné lieu à un réel transfert de compétences. Et cela, c'est génial. Deuxièmement, cette formation à distance a connu un faible taux d'abandon, inférieur à $20 \%$, ce qui s'explique par la taille restreinte des promotions (de vingt à trente participants), une approche collaborative, un tutorat de proximité et proactif et, en amont, une sévère sélection des candidats lors des entretiens d'admission.

\footnotetext{
${ }^{12}$ https ://www.univ-tIn.fr/-UFR-Ingemedia-.html
} 


\section{VIVIANE GLIKMAN et CATHIA PAPI : Toi personnellement, quelles ont été tes activités de recherche?}

DANIEL PERAYA : Personnellement j'ai participé à une quinzaine de projets européens visant à développer la formation à distance avec des financements de l'OFES de 1991 à 2012 dans le cadre des nombreux programmes (DELTA I et II, SIGMA, HUMANITIES I et II, SOCRATES, etc.). Plusieurs projets de recherche, de recherche-action ou de développement de dispositifs de formation à distance que j'ai coordonnés au nom de TECFA ont été financés par le FNS, notamment une participation importante dans le programme Campus virtuel suisse dont j'ai parlé. Ces projets ont largement contribué à développer l'expertise de TECFA dans le domaine de la formation à distance et, comme je le disais, à développer celle-ci en Suisse.

Ces projets financés sur fonds ont permis d'engager chaque fois plusieurs chercheurs et d'en stabiliser certains durant plusieurs projets. Ils ont donc bénéficié d'une formation et d'une expérience académiques et professionnelles importantes. Certains se sont intégrés au sein de TECFA, d'autres dans d'autres universités, hautes écoles pédagogiques, écoles secondaires, mais toujours dans le domaine des technologies éducatives.

\section{VIVIANE GLIKMAN et CATHIA PAPI : Quelles sont tes approches en termes de recherche et ont- elles évolué au cours des années?}

DANIEL PERAYA : Au départ, je suis issu du courant sémiopragmatique de l'analyse des médias dans leurs usages éducatifs. Mon centre d'intérêt principal est donc l'analyse des effets de ces dispositifs médiatiques sur les comportements cognitifs, mais aussi relationnels, socioaffectifs, et praxéologiques des apprenants. Votre question est d'autant plus importante pour moi que mon insertion dans une faculté de psychologie et des sciences de l'éducation, l'évolution de mes centres d'intérêt vers les formations entièrement ou partiellement à distance ont fait évoluer mes recherches vers de nouvelles thématiques, d'autres objets et terrains. Je répondrai en évoquant l'ouverture de l'analyse des représentations et de registres des représentations sémiocognitives à l'analyse de l'appropriation et des usages des TIC.

Cette évolution est cruciale pour deux raisons. II m'a paru essentiel de prendre en compte les effets des dispositifs médiatiques sur les comportements des enseignants autant que sur ceux des apprenants. Les premiers étaient en effet " oubliés " dans l'approche sémiopragmatique classique. Ensuite, il a été indispensable de situer et d'articuler l'approche sémiopragmatique initiale dans un cadre théorique plus large, d'autant que j'ai toujours considéré que les dispositifs de formation entièrement ou partiellement à distance sont un terrain privilégié pour étudier les processus de médiatisation et de médiation. L'élaboration progressive d'un cadre de référence relatif à la communication et à la formation médiatisées - les concepts de dispositif, de médiatisation et de médiation y jouent un rôle central - me semble la réponse, sans doute encore imparfaite, à cette exigence. Une seconde réponse renvoie à l'articulation entre les approches pédagogique et communicationnelle dont l'équilibre, l'histoire du domaine le montre, est souvent délicat à réaliser.

À titre d'exemple, je pourrais citer quelques recherches que je trouve emblématiques : a) l'analyse des formes de la communication éducative médiatisée dans le cadre d'un projet de recherche tessinois et plus particulièrement un modèle méthodologique d'analyse des rapports entre les textes et les paratextes, dont les représentations visuelles dans les sites Web étaient basées sur un modèle élaboré à partir de manuels scolaires d'économie et de biologie (Peraya et Nyssen,1995); b) le développement et l'instrumentation d'une procédure de réduction de la polysémie des icônes dans les logiciels et les environnements informatiques standard (Peraya et Ott, 2003); c) l'analyse des interactions verbales dans le cadre d'une 
classe virtuelle (Peraya et Dumont, 2003) utilisée dans le dispositif de formation STAF. II s'agissait d'analyser les effets du dispositif sur les formes de communication, sur la nature et la longueur des interactions, sur la réparation des rôles (apprenants, assistants et enseignants); d) enfin, récemment, une analyse exploratoire de certaines vidéos de MOOC (Campion, Peltier et Peraya, 2019) à partir d'une transposition au langage des vidéos de l'approche énonciative, des genres de textes et des types de discours (Bronckart, 1997).

\section{VIVIANE GLIKMAN et CATHIA PAPI : Comment vois-tu la place et le rôle de TECFA dans les années à venir? Penses-tu que la pandémie actuelle va avoir de l'importance dans son devenir et dans celui de la formation à distance en général?}

DANIEL PERAYA : En réalité, vous me posez deux questions. La première porte sur l'avenir de TECFA. J'ai quitté TECFA en 2014 et, depuis, il y a sans doute des tas de choses qui ont évolué et dont j'ignore les détails. J'ai une certitude : TECFA continue de se développer et de s'agrandir et je n'ai aucune crainte pour son avenir. Je ne vois pas ce qui, aujourd'hui, pourrait entraver son développement et son existence en tant que centre de référence dans le domaine.

La seconde question porte sur les effets de la pandémie sur la formation à distance. J'ajouterais sur l'hybridation des dispositifs de formation. J'y répondrai succinctement, bien que ce soit une question terriblement importante. La pandémie et la fermeture de tous les établissements de formation dans le monde constituent un évènement que l'on n'aurait jamais imaginé. Aussi n'a-t-on jamais autant parlé de formation à distance et d'hybridation. De nombreux enseignants et chercheurs ont écrit qu'il s'agissait d'une rare opportunité pour repenser le paradigme de l'enseignement et de l'apprentissage tandis que d'autres ont évoqué une occasion de faire évoluer radicalement les formes éducatives. Amaury Daele (2020), commentant les titres de la presse des journaux romands, écrivait récemment : " Dans 6 mois, 2 ans, l'enseignement supérieur sera hybride, titrent les journaux du jour. Pourrait-on dire que le virus a réussi à provoquer ce à quoi nous ne sommes pas arrivés depuis 25 ans et l'arrivée d'Internet dans les universités? ". Pas si sûr...

Je crains en effet que ce soit une arme à double tranchant. II me semble en effet que l'on a très mal parlé des formations à distance et des dispositifs hybrides de formation. On a fait passer pour de la formation à distance la simple mise en ligne de ressources pédagogiques (documents de cours au format PDF ou PowerPoint), des enregistrements de cours donnés devant un auditoire vide. Au mieux, certains cours ont été donnés en synchrone dans un dispositif de visioconférence. Quant au " dispositif hybride ", il est devenu une simple modalité organisationnelle, l'alternance entre les moments de présence et ceux passés à distance. Mais est-ce bien ce dont nous parlons depuis le début de cet entretien? Est-ce bien cette conception uniquement substitutive que nous défendons? Je ne crois pas. Je peux comprendre que lors du premier confinement, dans l'urgence et l'impréparation de la majorité des enseignants, cela a été le cas (DMS, 2020). Mais, depuis la fin de cette première période de confinement, plutôt que de promouvoir une réflexion pédagogique de fond, formation à distance et dispositifs hybrides sont devenus des slogans derrière lesquels se cachent des enjeux politiques.

Aussi, je crois que ce qui s'est pratiqué le plus souvent dans l'urgence et ce qui se pratique malheureusement encore aujourd'hui sous le couvert de cette terminologie n'ont pas grand-chose à voir avec ce que sont la formation à distance et les dispositifs hybrides de formation que nous sommes nombreux à défendre. L'insatisfaction des étudiants et des enseignants par rapport à ces expériences imparfaites pourrait laisser des traces négatives, ternir l'image de la formation à distance et détourner, à l'avenir, les uns et les autres de ce type de formation. 
De plus, les incertitudes de la situation, son inscription dans la durée et le manque de perspective pourraient, selon moi, amener un autre risque, celui d'une erreur de jugement qui consisterait à attribuer aux modalités actuelles de formation cet état de souffrance socioaffective que vivent beaucoup de nos élèves et étudiants. Si celui-ci est bien réel, c'est la pandémie et la situation sanitaire qui en sont la cause. D'ailleurs, de nombreux adultes en souffrent aussi. Formation à distance et hybridation participent de ce mal être, mais n'en sont pas la cause principale. Or ce raccourci, qui consiste à prendre un facteur aggravant pour la cause principale, est tentant et on l'entend déjà dans certains discours du grand public.

II y a donc un énorme travail de documentation, de recherche, de dissémination et de formation pour prévenir ces effets potentiels, mais pervers, de la crise sanitaire.

\section{Liste de références}

Attwell, G. (2006). Personal Learning Environments-the future of eLearning? eLearning Papers, 2(1). https://citeseerx.ist.psu.edu/viewdoc/summary?doi=10.1.1.97.3011

Bernasconi, L. (2009). Présence de l'enseignant dans le dispositif « double hybride »MALTT@DIS : une étude exploratoire [Mémoire de Master MALTT]. TECFA, Université de Genève.

Bonfils, P. et Peraya, D. (2011). Environnements de travail personnels ou institutionnels? Les choix d'étudiants en ingénierie multimédia à Toulon. Dans L. Vieira, C. Lishou et N. Akam (dir.), Le numérique au cœur des partenariats : Enjeux et usages des technologies de l'information et de la communication (p. 13-28). Presses universitaires de Dakar. https://archive-ouverte.unige.ch/unige:17874

Bronckart, J.-P. (1997). Activité langagière, textes et discours : pour un interactionisme socio-discursif. Delachaux et Niestlé.

Campion, B., Peltier, C. et Peraya, D. (2019). Analyse communicationnelle de vidéos de Moocs : quelle magistralité les choix discursifs construisent-ils? Éducation et formation, e-313, 75-92. http://revueeducationformation.be/index.php?revue=33\&page $=3$

Daele, A. (2020, 7 décembre). Groupe « Pédagogie universitaire » [page Facebook]. Facebook. https://www.facebook.com/groups/pedagogieuniversitaire

DMS. (2020). Débat-discussions, Distances et médiations des savoirs, (29, 30, 31, 32). https://journals.openedition.org/dms/

Joye, F., Deschryver, N. et Peraya, D. (2003). Comment développer un campus virtuel? Dans B. Charlier et D. Peraya (dir.), Technologie et innovation en pédagogie : dispositifs innovants de formation pour l'enseignement supérieur (p. 93-102). De Boeck.

Lebrun, M., Peltier, C., Peraya, D., Burton, R. et Manusco, G. (2014). Un nouveau regard sur la typologie des dispositifs hybrides de formation. Propositions méthodologiques pour identifier et comparer ces dispositifs. Éducation et Formation, e-301, 55-74. http://revueeducationformation.be/index.php?revue=19\&page=3

Lifländer, V. P. (1993). Project Computer-Aided Learning at Helsinki University of Technology 1991-1993. Dans M. Linna et P.Ruotsala (dir.), Proceedings of Hypermedia in Vaasa '93 (209-212). Vaasa Institute of Technology.

Lombardi, E. (2007). Analyse communicationnelle des effets cognitifs d'un dispositif éducatif médiatisé. Le cas de la médiatisation d'un cours $3 D$ en images virtuelles immersif et interactif et ses impacts sur la mémoire explicite. [Thèse de doctorat]. Université du Sud Toulon-Var, France.

Peraya, D. et Bonfils, P. (2012). Nouveaux dispositifs médiatiques : comportements et usages émergents. Le cas d'étudiants toulonnais en formation à l'UFR Ingémédia. Distances et Médiations des Savoirs, (1). https://journals.openedition.org/dms/126

Peraya, D. et Collard, A. S. (2008). La métaphore dans les campus virtuels : des formes de la représentation à la « représent-action ». Recherches en communication, (29), 57-80. 
Peraya, D., Depover, C. et Jaillet, A. (2013). Un master à distance pour une formation aux technologies éducatives : le diplôme UTICEF - ACREDITÉ. Dans P.-J. Loiret (dir.), Un détour par le futur. Les formations ouvertes et à distance à l'Agence universitaire de la francophonie. 1992-2012 (p. 83-102). Agence universitaire de la francophonie, Archives contemporaines. http://www.bibliotheque.auf.org/doc num.php?explnum id=822

Peraya, D. et Dumont, P. (2003). Interagir dans une classe virtuelle : analyse des interactions verbales médiatisées dans un environnement synchrone. Revue française de pédagogie, 145, 51-61. https://www.persee.fr/doc/rfp_0556$\underline{78072003 \text { num } 14512984}$

Peraya, D. et Nyssen, M.C. (1995). Les paratextes dans les manuels scolaires de biologie et d'économie : une étude comparative. Faculté de psychologie et des sciences de l'éducation de l'Université de Genève.

Peraya, D. et Ott, D. (2003). Analyse de quelques stratégies de lecture des pages Web : une approche sémiocognitive du traitement des unités d'information dans les sites Web. Recherches en communication, 19, 103-112. https://archive-ouverte.unige.ch/unige: 17674

Peraya, D. et Peltier, C. (2012). Typologie des dispositifs hybrides : configurations et types. Dans N. Deschryver et B. Charlier (dir.), Dispositifs hybrides, nouvelle perspective pour une pédagogie renouvelée de l'enseignement supérieur. Rapport final (p. 54-86). http://prac-hysup.univ-lyon1.fr/spiralfiles/download?mode=inline\&data $=1757974$

TECFA (1999). Rapport d'activités 1989-1999. http://tecfa.unige.ch/tecfa/general/tecfa-report-99.html

Vincent, G. (1980). L'école primaire française. Étude sociologique. Presses universitaires de Lyon/Éd. de la Maison des sciences de l'homme. https://presses.univ-lyon2.fr/product/show/lecole-primaire-francaise/537

Vincent, G. (2008). La socialisation démocratique contre la forme scolaire. Éducation et francophonie, 36(2), 47-62. https://www.erudit.org/en/journals/ef/2008-v36-n2-ef2872/029479ar/abstract/ 